\title{
VARIACIONES SOBRE TEMAS DE CARLOS ALCHOURRÓN Y EUGENIO BULYGIN. DERROTABILIDAD, LAGUNAS AXIOLÓGICAS E INTERPRETACIÓN ${ }^{1}$
}

\author{
RICCARDO GUASTINI $^{2}$ \\ UNIVERSIDAD DE GÉNOVA - ITALIA
}

\section{RESUMEN:}

El trabajo pretende analizar las relaciones entre la derrotabilidad de las normas y las lagunas axiológicas desde la perspectiva de una teoría realista de la interpretación. Algunos ejemplos muestran que los dos conceptos denotan fenómenos que se presentan a lo largo del proceso de interpretación y que dependen de las estrategias interpretativas de los juristas. El análisis de los ejemplos permite desprender algunas conclusiones: entre otras, la siguientes:

1. La derrotabilidad no es una propiedad objetiva de las normas, antecedente a toda interpretación: por el contrario, es una de sus posibles consecuencias, es el resultado de una operación interpretativa (en particular, de una interpretación no literal, sino restrictiva, por medio de la cual se crean excepciones implícitas).

1 Artículo publicado en DOXA, Cuadernos de Filosofía del Derecho, 31 (2008) ISSN: 0214-8676 pp. 143-155

2 Profesor emérito de Filosofía del Derecho en la Universidad de Génova - Italia ORCID: 0000-0002-2125-8196 
2. Al derrotar una norma se excluyen de su alcance algunos supuestos (que, sin embargo, de acuerdo con una distinta interpretación sí estarían reglados por la norma); si dichos supuestos no resultan reglados por otra norma del sistema, el sistema está lagunoso; entonces, la derrota produce una laguna.

3. Dicha laguna puede ser considerada axiólogica o normativa desde perspectivas interpretativas diferentes. Es decir, no se pueden distinguir las lagunas normativas de las lagunas axiológicas independientemente de la interpretación. Entonces incluso las lagunas normativas son variables dependientes de la interpretación: según una interpretación determinada hay una laguna normativa, mientras que según otra interpretación esta laguna milagrosamente desaparece, no subsiste en tanto laguna normativa, y entonces se convierte en laguna axiológica.

4. La derrotabilidad de las normas y las lagunas axiológicas dependen no de la presencia, en el sistema jurídico, de principios, como suponen algunos autores, sino de las valoraciones de los intérpretes, que a menudo toman la forma de «teorías» jurídicas o tesis dogmáticas: algo construido por los juristas antes e independientemente de la interpretación de uno u otro enunciado normativo específico.

\section{PALABRAS CLAVE:}

Derrotabilidad de las normas, lagunas axiológicas, teoría realista de la interpretación. 


\section{ABSTRACT:}

The paper aims at analysing the relationships between defeasibility of norms and axiological gaps from the standpoint of a skeptical theory of legal interpretation. A number of examples show that the two concepts denote phenomena, which arise along the process of interpretation, and depend on the interpretive strategies of jurists. The analysis of such examples allows for a number of conclusions, such as the following.

1. Defeasibility is not an objective property of norms, prior to interpretation; on the contrary, it is one of the possible consequences of interpretation - it is the result of a particular interpretive act (viz., a non-literal, restrictive interpretation through which implicit exceptions are created).

2. By defeating a norm, interpreters exclude from its domain of application certain facts, (which according to a different interpretation would be included). Whenever such facts are not regulated by other valid norms, there is a gap in the legal system. Thus, the defeat produces a gap.

3. Such a gap may be considered either normative or axiological from different interpretive standpoints. This means that one cannot distinguish between normative and axiological gaps independently of interpretation. As a consequence, even normative gaps depend on interpretation: according to a certain interpretation, a normative gap exists, while according to a different interpretation this gap disappears, does not exist as a normative gap, and, therefore, it converts itself in axiological. 
4. Defeasibility of norms and axiological gaps do not depend on the existence of principles (as many author maintained), they simply depend on interpreters' evaluations, which often appear under the clothes of legal theories or dogmatic theses built up by jurists before and independently of the interpretation of any particular legal text.

\section{KEYWORDS:}

Defeasibility of norms, axiological gaps, the sceptical theory of legal interpretation. 


\section{INTRODUCCIÓN}

Lo que quiero hacer en estas pocas páginas es intentar conectar sistemáticamente dos ideas que se encuentran en la teoría del Derecho de C. Alchourrón y E. Bulygin -la de laguna axiológica y la de derrotabilidad de las normas- con una teoría realista de la interpretación ${ }^{3}$.

1. 1. Laguna axiológica. Una laguna axiológica es una situación en la cual un cierto supuesto de hecho sí está reglado por una norma, pero -según la opinión del intérprete- está reglado de forma axiológicamente inadecuada [...], porque el legislador no tuvo en cuenta una distinción que debería haber tomado en cuenta. Se supone que el legislador no ha tenido en cuenta la distinción en cuestión por no haberla previsto y que, de haberla considerado, hubiera dado una solución diferente al supuesto de que se trata ${ }^{4}$.

3 Las ideas fundamentales de dicha teoría se encuentran en: TARELLO, G. L'interpretazione della legge. Milano, 1980; GuAstinI, R. L'interpretazione dei documenti normativi. Milano, 2004; CHIASSONI, P. "Codici interpretativi. Breviario metodologico per aspiranti giuristi", en Comanducci, P., y GuAstinI, R. (eds.). Analisi e Diritto 2002-2003. Ricerche di giurisprudenza analitica. Torino, 2004. Cfr. también DiciotTI, E. Interpretazione della legge e discorso razionale. Torino, 1999 .

4 En mi exposición prefiero evitar la terminología técnica introducida por Alchourrón y Bulygin (tesis de relevancia, hipótesis de relevancia, etc.) para que lo que digo resulte fácilmente comprensible a cualquier jurista, que no haya leído su libro. Alchourrón, C. E., y BULYGIN, E. Introducción a la metodología de las ciencias jurídicas y sociales. Buenos Aires, 1974 p. 158. 
Dicho de otra forma, una laguna axiológica no es un caso sin solución, sino más bien un caso con una mala solución ${ }^{5}$. Evidentemente, la laguna aquí no consiste en la falta de una norma sin más. Lo que falta no es una norma que regule el supuesto en cuestión, porque ese supuesto en efecto está reglado (de no ser así, habría una laguna no axiológica, sino normativa). Lo que falta es una norma satisfactoria o justa y, más precisamente, una norma diferenciadora, es decir una norma que regule de forma distinta un supuesto que al intérprete le parece distinto $^{6}$.

1.2. Derrotabilidad de las normas. Cualquier norma jurídica puede ser reconstruida como un enunciado condicional: Si H, entonces $J$, donde el antecedente denota un clase de supuestos de hecho y el consecuente denota una clase de consecuencias jurídicas (tales como: obligaciones, permisiones, prohibiciones, sanciones, validez o invalidez de actos, etc. ${ }^{\text {. }}$

5 Navarro, P., y Rodríguez, J. “Derrotabilidad y sistematización de normas jurídicas", en Isonomía. N. ${ }^{\circ} 13,2000$, p. 76.

6 Aquí no me ocupo de otro concepto de laguna axiológica, que ha sido elaborado por RodríGUEZ, J. Lógica de los sistemas jurídicos. Madrid, 2002, p. 76 y ss.; cfr. también BuLYGIN, E. “En defensa de El Dorado. Respuesta a Fernando Atria", en AtRiA, F., et al. Lagunas en el Derecho. Madrid-Barcelona, 2005.

RatTI, B. "Dos modelos de relevancia normativa", en Seminario Homenaje a Eugenio Bulygin. Barcelona, Universidad Pompeu Fabra, junio, 2006. (inédito)

7 Este concepto de norma es un poco mas amplio de lo adoptado por. Alchourrón, C. E. y Bulygin, E. en Introducción a la metodología de las ciencias jurídicas y sociales, incluyendo enunciados que sólo expresan normas, en el sentido que pertenecen a un sistema 
Ahora bien, muchas (sino todas) la formulaciones normativas [que se encuentran en un ordenamiento jurídico] son derrotables, esto es, poseen usualmente excepciones implícitas, existen circunstancias que derrotan la norma aunque ellas no están explícitamente enunciadas ${ }^{8}$, circunstancias que desplazan la obligación, aunque no estén formuladas expresamente ${ }^{9}$. La derrotabilidad implica dos consecuencias muy importantes. En primer lugar, para el condicional superable [i.e., derrotable] no vale la ley del refuerzo del condicional [...]. En segundo lugar, tampoco vale la ley del modus ponens ${ }^{10}$.

normativo, aunque no tienen fórmulas deónticas en el consecuente. Alchourrón, C. E., y Bulygin, E. Introducción a la metodología de las ciencias jurídicas y sociales.

8 Alchourrón, C. E. "Sobre Derecho y lógica", en Isonomía. N. o 13, 2000, p. 24.

9 Alchourrón, C. E., y Bulygin, E. "Norma jurídica”, en Garzón VAldés, E., y LAPORTA, F. J. (eds.). El Derecho y la justicia. Madrid, Enciclopedia Iberoamericana de Filosofía, N. ${ }^{\circ} 11$, 1996, p. 146. Otros sentidos de derrotabilidad son examinados por REDONDO, M. C. "Teorías del Derecho e indeterminación normativa", en Doxa. N. ${ }^{\circ}$ 20, 1997; y en RodríGuEZ, J., en su libro Lógica de los sistemas jurídicos, en su capítulo IV, en particular, me parece importante señalar que la derrotabilidad, en el sentido de que una norma sería sujeta no a excepciones implícitas, sino a las excepciones que otras normas expresas del mismo sistema normativo introducen en ella, nada tiene que ver con el concepto de derrotabilidad que nos interesa en este contexto. Cfr. RodríguEZ, J. Lógica de los sistemas jurídicos, p. 358, 364 y ss.

10 La lógica de los condicionales derrotables ha sido elaborada por Alchourrón, C. E. en varios ensayos técnicos, que no es preciso mencionar en este contexto. Alchourrón, C. E., y Bulygin, E. "Norma jurídica", op. cit., p. 145 y ss. 
Dicho de otra forma, una norma derrotable es una norma sujeta a excepciones implícitas que no pueden ser enumeradas exhaustivamente de antemano, de manera que no es posible precisar por anticipado las circunstancias que operan como genuina condición suficiente de su aplicación ${ }^{11}$.

Para mostrar la conexión entre lagunas axiológicas, derrotabilidad, e interpretación vamos a ver unos ejemplos ficticios (aunque no completamente ficticios).

\section{ALGUNOS EJEMPLOS}

2.1. Primer ejemplo. En una república con constitución rígida, forma parlamentaria de gobierno, y control concentrado a posteriori sobre la constitucionalidad de las leyes, una disposición constitucional establece: $E l$ Presidente de la República puede oponer su veto a la promulgación de las leyes.

¿A cuáles leyes se refiere esta disposición? Según la interpretación literal, a cualquier ley, porque el texto no distingue de ninguna forma entre leyes de tipos diferentes.

Ahora, imaginemos un jurista que argumenta más o menos de la manera siguiente:

11 Rodríguez, J. "Derrotabilidad e indeterminación del Derecho. Respuesta a JuAn CARlos BAYÓN", en BAYÓN, J. C., y RodrígueZ, J. Relevancia normativa en la justificación de las decisiones judiciales. Santa Fe de Bogotá, Universidad Externado de Colombia, 2003, p. 210. 
a) Cabe distinguir entre dos tipos de leyes: por un lado, las leyes ordinarias; por otro lado, las leyes de revisión constitucional (esto es lo que, en teoría de la interpretación, se llama la técnica de la disociación).

b) En el ordenamiento vigente (gobierno parlamentario) el Presidente de la República no es el jefe del poder ejecutivo, sino un poder neutro -algo como el pouvoir neutre de B. Constant- que tiene funciones no políticas, sino sólo de garantía de la constitución (esta, nótese, es una tesis no estrictamente interpretativa, sino dogmática: una tesis de teoría constitucional, si se quiere decirlo así).

c) La función del veto presidencial es la de permitir al Presidente un control previo (distinto de lo del Tribunal Constitucional, que es sucesivo) sobre la constitucionalidad de las leyes; en particular, el Presidente tiene el poder de oponer su veto frente a leyes cuya inconstitucionalidad es evidente (esta es una tesis dogmático-interpretativa sobre la ratio legis, o sea sobre la finalidad de la disposición que se comenta).

d) Por otro lado, un problema de constitucionalidad sólo puede presentarse con respecto a las leyes ordinarias: una ley de revisión constitucional nunca puede ser inconstitucional (excepto por razones formales), porque las leyes de revisión consisten precisamente en un cambio del texto constitucional. Las leyes de revisión, se podría decir, siempre, por definición, son inconstitucionales, pero están autorizadas a serlo (esta 
tesis se sigue analíticamente desde el concepto común de revisión constitucional).

e) Entonces, la disposición constitucional de que se trata debe ser interpretada en el sentido que se refiere sólo a las leyes ordinarias, no a las leyes de revisión (esta es una primera conclusión interpretativa, que se sigue de la disociación combinada con unas tesis dogmáticas).

f) La constitución no dice nada en absoluto sobre el poder presidencial de veto en relación con las leyes de revisión (se trata de una segunda conclusión interpretativa que, como la precedente, se sigue de la disociación y de la dogmática constitucional).

g) Por consecuencia, el Presidente de la República no tiene el poder de oponer su veto a las leyes de revisión (esta es una segunda conclusión interpretativa, en sentido amplio, extraída a contrario a partir del silencio del texto constitucional: inclusio unius est exclusio alterius).

Esta forma de razonamiento, como se ve, produce consecuencias de gran interés.

1. En primer lugar, mediante la técnica de la disociación, nuestro jurista imaginario crea una excepción implícita en la disposición constitucional interpretada. Es decir, la hace una disposición derrotable o, mejor dicho, la derrota.

2. En segundo lugar, el brinda, de dicha disposición, una interpretación restrictiva: el alcance de la disposición resulta restringido a las leyes ordinarias (mientras 
que, según una interpretación literal, ella se aplicaría a todas las leyes sin distinción).

3. En tercer lugar, produce una laguna: la disposición constitucional, reglando sólo el supuesto ley ordinaria, no regula las leyes de revisión; el supuesto ley de revisión (en relación con el poder presidencial de veto) no está reglado por la constitución de ninguna forma. Para quien interpreta literalmente la disposición de que se trata, por el contrario, esta laguna no es normativa, sino axiológica, pues el texto se refiere a las leyes sin distinción, y entonces también a las leyes de revisión.

4. En cuarto lugar, nuestro jurista colma la laguna que el mismo ha creado por una norma implícita (en sentido amplio, no en sentido lógico) -una norma de creación doctrinal (Juristenrecht)- según la cual el Presidente no tiene poder de veto sobre las leyes de revisión.

2.2. Segundo ejemplo. En el mismo ordenamiento constitucional que antes, donde el Gobierno tiene el poder de promulgar, en ciertas circunstancias, actos legislativos, que (como las leyes stricto sensu) pueden ser sometidos al control sucesivo del Tribunal Constitucional, una disposición constitucional establece: El Tribunal de Cuentas ejerce el control de legitimidad sobre los actos del Gobierno.

¿A cuáles actos se refiere esta disposición? Según la interpretación literal, a cualquier acto del gobierno, evidentemente, porque el texto no distingue de ninguna forma entre actos de tipos diferentes. 
Sin embargo, imaginemos un jurista que argumenta, más o menos, de la forma siguiente.

a. En nuestro ordenamiento existen dos tipos de actos de gobierno: actos legislativos (con fuerza de ley) y actos no legislativos (reglamentarios y administrativos).

b. Legitimidad puede significar, a la vez, conformidad con las leyes stricto sensu (legalidad) o bien conformidad con la constitución (legitimidad constitucional o constitucionalidad).

c. Los actos de valor legislativo, evidentemente, no tienen que ser conformes a las leyes (por el contrario, pueden derogar a las leyes: en eso precisamente consiste su valor legislativo). Los actos legislativos sólo deben ser conformes a la constitución.

d. Sin embargo, en nuestro ordenamiento, el control de conformidad con la constitución sobre los actos de valor legislativo es competencia (que se supone exclusiva) del Tribunal Constitucional.

e. Por tanto, la disposición constitucional de que se trata debe ser interpretada en el sentido que se refiere sólo a los actos no legislativos; los actos legislativos quedan fuera de su ámbito de aplicación.

f. La constitución no dice nada sobre la competencia del Tribunal de Cuentas sobre los actos legislativos del Gobierno. 
g. Por consecuencia, el Tribunal de Cuentas no tiene competencia para controlar la legitimidad de los actos legislativos del Gobierno.

Como en el caso precedente, nuestro jurista imaginario ha hecho cuatro cosas importantes:

1. Por medio de la técnica de la disociación, ha derrotado la disposición constitucional, introduciendo en ella una excepción implícita: es decir, una excepción que, según la interpretación literal, no existe en absoluto.

2. De esta forma ha propuesto una interpretación restrictiva de dicha disposición.

3. Al mismo tiempo ha creado una laguna: la constitución no regula los poderes del Tribunal de Cuentas sobre los actos legislativos del Gobierno. Por supuesto, a quien interpreta literalmente, esta laguna le parece no normativa -pues el texto se refiere a todos los actos gubernativos- sino axiológica.

4. Y por último nuestro jurista ha colmado la laguna con la norma de creación doctrinal según la cual el Tribunal de Cuentas no es competente para controlar la legitimidad de los actos legislativos del Gobierno.

2.3. Tercer ejemplo. En el mismo ordenamiento constitucional, una disposición de la Constitución Política del Estado establece: Ningún acto del Presidente de la República es válido si no está refrendado por el ministro proponente. 
Interpretación literal: cualquier acto presidencial debe ser refrendado por el ministro proponente. Esto supone que por cualquier acto presidencial haya un ministro proponente, y entonces una propuesta ministerial. Es decir, el Presidente no puede promulgar ningún acto sino sobre la base de una propuesta ministerial.

Pero imaginemos un jurista que argumenta, más o menos, de la forma siguiente.

a) Dentro de una forma de gobierno parlamentaria, el Presidente de la República no es el jefe del poder ejecutivo: es un poder neutro con funciones de garantía de la Constitución Política (se trata de una premisa dogmática que ya conocemos).

b) Por tanto existen actos que el Presidente cumple en el ejercicio de su función garantista: dichos actos no tienen contenido político partidario, son políticamente imparciales, y entonces no presuponen -no requierenninguna propuesta ministerial.

c) Por consecuencia, hay que distinguir entre dos tipos de actos presidenciales: los actos sustancialmente gubernativos (cumplidos sobre la base de una propuesta ministerial) y los actos «estrictamente» presidenciales (sin propuesta ministerial).

d) Ahora bien, la disposición constitucional que se comenta se refiere no a todos los actos del Presidente de la República, sino sólo a los actos sustancialmente 
gubernativos; los otros actos presidenciales quedan fuera de su alcance.

e) Entonces, los actos estrictamente presidenciales no están reglados por la Constitución Política.

f) Porconsecuencia,los actos estrictamente presidenciales no requieren ningún refrendo ministerial (o, quizás, requieren el refrendo no del ministro proponente, que simplemente no está, sino del ministro competente por materia).

Una vez más, nuestro jurista imaginario ha hecho cuatro cosas:

1. Ha derrotado la disposición constitucional, introduciendo en ella una excepción implícita, que, según la interpretación literal, no subsistiría.

2. De esta forma ha propuesto una interpretación restrictiva de dicha disposición.

3. La interpretación restrictiva le ha permitido identificar una laguna: la constitución no regula los actos estrictamente presidenciales en relación con el refrendo ministerial. A quien interpreta literalmente, tal laguna le parece evidentemente axiológica, pues el texto constitucional no distingue, y se refiere a los actos presidenciales sin más.

4. Por último, nuestro jurista ha colmado esta laguna con la norma de creación doctrinal, según la cual los actos 
estrictamente presidenciales no requieren refrendo ministerial.

\section{ALGUNAS CONCLUSIONES}

No sería difícil multiplicar los ejemplos. Pero, ¿estos simples ejemplos se pueden generalizar? Yo creo que sí. De estos ejemplos, si bien (más o menos) ficticios, se desprenden varias consecuencias interesantes.

1. Los ejemplos sugieren que los conceptos de laguna axiológica y de derrotabilidad pertenecen, para decirlo así, no a la teoría de los sistemas normativos, sino más bien a la teoría de la interpretación. Denotan fenómenos que se presentan a lo largo del proceso de interpretación y que dependen de las estrategias interpretativas de los juristas (o de los jueces, por supuesto). Tal vez, hace falta recordar que las actividades de sistematización del Derecho siguen, no preceden, las decisiones interpretativas: no se hacen inferencias desde los textos (todavía no interpretados), sino sólo desde los significados, que, precisamente, presuponen la interpretación.

2. Muchas veces lagunas axiológicas y derrotabilidad son, por decirlo así, las caras de una misma medalla ${ }^{12}$. Al derrotar una norma se excluyen de su alcance algunos supuestos (que, sin embargo, de acuerdo con una distinta interpretación sí estarían reglados por la norma). A veces

12 Rodríguez, J. Lógica de los sistemas jurídicos. op.cit., p. 377; Ídem., y BAYÓN, J. C. "Derrotabilidad e indeterminación del Derecho. Respuesta a JUAN CARLOS BAYÓN", op. cit., p. 229. 
dichos supuestos resultan reglados por otra norma del sistema, pero otras veces resultan no reglados por ninguna norma perteneciente al sistema. En este último caso el sistema está lagunoso. Entonces, la derrota produce una laguna ${ }^{13}$.

13 La derrota produce lagunas, pero no todas las lagunas se producen por derrota. Por ejemplo, en la práctica de algunos tribunales constitucionales se encuentran los razonamientos siguientes.

El legislador, al regular una clase de supuestos (por ejemplo, las «grandes empresas»), ha omitido regular de la misma manera otra clase de supuestos (por ejemplo, las "pequeñas empresas») que, según el juez constitucional, es «sustancialmente» igual a la clase de supuestos reglada.

El legislador, al regular una clase de supuestos (por ejemplo, las «empresas» sin distinción), ha omitido distinguir, dentro de esa clase, dos subclases (por ejemplo, las grandes y las pequeñas empresas) que, según el juez constitucional, son sustancialmente distintas y, por tanto, exigen regulaciones distintas.

En ambos casos, hay una laguna axiológica (de un tipo particular: Cfr. PARODI, G. "Lacune e norme inespresse nella giurisprudenza costituzionale", en ComanducCI, P., y GuAstini, R. (eds.). Struttura e dinamica dei sistemi giuridici. Torino, 1996; Ídem. La sentenza additiva a dispositivo generico. Torino, 1996, p. 131 y ss.; el primer caso, sin embargo, no sería un caso de laguna axiológica. Véase ALCHOURRÓN, C. E., y BULYGIN, E. Introducción a la metodología de las ciencias jurídicas y sociales. p. 160). En ambos casos, la ley se considera inconstitucional, en razón de la laguna axiológica que contiene. La existencia de la laguna se argumenta sobre la base del principio constitucional de igualdad, entendido en el sentido de que los casos iguales deben ser tratados de la misma forma y los casos distintos deben ser tratados de forma distinta. Pero, en el primer caso (laguna axiológica sin derrota), lo que falta, según el juez constitucional, es una norma igualadora; en el segundo caso (laguna axiológica como consecuencia de la derrota), lo que falta, en cambio, es una norma diferenciadora. En ambos casos, el Tribunal Constitucional emite una sentencia aditiva. Cfr. MORESO, J. J. La indeterminación del Derecho y la interpretación de la Constitución. Madrid, 1997, p. 171 y ss. 
3. El concepto de laguna axiológica no pertenece al lenguaje de los juristas (o de los jueces): pertenece al lenguaje de la teoría del Derecho, más precisamente de la metajurisprudencia ${ }^{14}$, es decir, del análisis lógico del discurso de los juristas (y de los jueces).

Quiero decir que quien afirma la existencia de una laguna -salvo que hable de jure condendo, criticando el sistema jurídico vigente ${ }^{15}$ - nunca admite que se trata de una laguna axiológica: por el contrario, siempre sugiere que dicha laguna es auténticamente normativa, es decir es un defecto objetivo del sistema normativo, independiente de cualquier valoración ${ }^{16}$. Sin embargo, para decir a nivel de metalenguaje -o sea desde el punto de vista externo- que la laguna en cuestión no es normativa, sino axiológica, debemos presuponer una interpretación del mismo texto, distinta de la interpretación propuesta por quien afirma la existencia de dicha laguna.

La lagunas axiológicas, entonces, parecen depender de la interpretación dos veces, o en doble sentido. Por un lado, dependen de la interpretación en el sentido que quien afirma la existencia de una laguna axiológica presupone una determinada interpretación -normalmente restrictiva-

14 Bоввіо, N. "Essere e dover essere nella scienza giuridica", en Ídem. Studi per una teoria generale del Diritto. Torino, 1970.

15 Rodríguez, J. Lógica de los sistemas jurídicos. op.cit., p. 82.

16 Creo que tiene razón Rodríguez, J. al sugerir que, a menudo (o quizás normalmente), los enunciados de los juristas que afirman la existencia de una laguna (axiológica), son simplemente enunciados interpretativos y no enunciados de crítica ético-política del sistema jurídico vigente. RODRíGUEZ, J. "Lagunas axiológicas y relevancia normativa, en Doxa. N. ${ }^{\circ}$ 22, 1999. 
del texto. Por otro lado, dependen de la interpretación en el sentido que quien afirma, a nivel de metalenguaje, que dicha laguna es precisamente axiológica, y no normativa, presupone a su vez una interpretación distinta -normalmente literal- del mismo texto.

Y esto tiene una consecuencia tal vez aún más inquietante. A veces se habla como si las lagunas axiológicas dependiesen de las valoraciones de los intérpretes, mientras que las lagunas normativas serían algo como propiedades objetivas del Derecho. De hecho, no es así. Si es verdad que no se pueden distinguir las lagunas normativas de las lagunas axiológicas independientemente de la interpretación, se sigue que una cierta laguna puede ser considerada normativa o axiológica desde puntos de vistas interpretativos distintos ${ }^{17}$. Pero esto quiere decir que incluso las lagunas normativas son variables dependientes de la interpretación: según una interpretación determinada hay una laguna normativa, mientras que según otra interpretación esta laguna milagrosamente desaparece, no subsiste en tanto laguna normativa, y entonces se convierte en laguna axiológica ${ }^{18}$.

17 Cfr. Redondo, M. C. "Reglas genuinas y positivismo jurídico", en Comanducci, P., y Guastini, R. (eds.). Analisi e Diritto. Ricerche di giurisprudenza analitica. Torino, 1998, p. 256.

Una disposición que resuelve un caso genérico bajo cierta interpretación puede constituir una laguna en una interpretación diferente; un sistema incompleto (con lagunas) puede ser transformado, mediante la interpretación [...] en un sistema completo (sin lagunas). AlCHOURRÓN, C. E. "Sobre Derecho y lógica", op.cit., p. 21.

Ídem., y BuLYGIN, E. Introducción a la metodología de las ciencias jurídicas y sociales. op.cit., p. 144. 
4. Los conceptos de derrotabilidad y de laguna axiológica son nuevos (o relativamente nuevos) en el lenguaje de la teoría del Derecho, pero ambas cosas son, a menudo, el resultado de una forma de interpretación bien conocida: la interpretación restrictiva, o sea aquella interpretación que reduce el alcance de una norma.

Por otra parte, el concepto de interpretación restrictiva es, evidentemente, un concepto de relación: ¿restrictiva con respecto a qué? Para afirmar que una interpretación es restrictiva hace falta presuponer una interpretación distinta: normalmente, una interpretación literal. Nótese, sin embargo, que incluso la interpretación literal es una interpretación, tal vez menos discutible, pero -en tanto interpretación- no más objetiva o neutral que otras interpretaciones posibles.

La interpretación restrictiva, como cualquier interpretación no literal, supone, expresa o tácitamente, el argumento (retórico) de la ratio legis, o sea de la intención -a menudo contrafáctica- de la autoridad normativa ${ }^{19}$. Más precisamente, quien rechaza la interpretación literal supone: primero, que existe una discrepancia entre lo que la autoridad normativa ha dicho y lo que quería decir (o hacer); segundo, que la intención tiene que prevalecer sobre el texto $^{20}$. Esta última suposición, evidentemente, no es otra cosa que una ideología política. Por otra parte, les enunciados sobre la intención de las autoridades normativas no pueden seriamente ser considerados como enunciados empíricos: no existe acceso a la mente de un Legislador, sobre todo si es un órgano colegiado ${ }^{21}$.

\footnotetext{
19 Guastini, R. L'interpretazione dei documenti normativi. p. 142 y ss.

20 Schauer, F. Playing by the Rules. p. 74 y ss.

21 Alchourrón, C. E. “Sobre Derecho y lógica”, op. cit., p. 23, 27 y ss.
} 
5. La interpretación restrictiva es el resultado de una técnica interpretativa específica: la técnica de la disociación ${ }^{22}$, algo parecido al distinguishing de los jueces de Common Law.

Esta técnica consiste en introducir en las normas distinciones nuevas, es decir no hechas por la autoridad normativa; consiste en descomponer la clase de los supuestos previstos por el texto -según la interpretación literal- en dos (o más) subclases, con el intento de sugerir que dichas subclases, siendo sustancialmente distintas, no pueden no tener consecuencias jurídicas diferentes ${ }^{23}$. La disociación, precisamente, es el instrumento argumentativo que permite crear lagunas axiológicas y derrotar las normas.

6. La derrotabilidad no es, como a veces se piensa, una peculiaridad de los principios $^{24}$. Es posible que los principios sean intrínsecamente derrotables -en el sentido que la derrotabilidad forma parte del propio concepto de principio- pero lo que es cierto es que cualquier norma puede ser convertida en derrotable: de hecho, los juristas lo hacen continuamente.

22 Perelman, Ch. y Olbrechts-Tyteca, L. Trattato dell'argomentazione. cap. IV. Torino, 1966.

23 GuASTINI, R. L'interpretazione dei documenti normativi. p. 163 y ss.; DiciotTI, E. Interpretazione della legge e discorso razionale. p. 451 y ss.

24 DwORKIN, R. Taking Rights Seriously. 2. ${ }^{\text {a }}$ ed. London, 1978; GIANFORMAGGIO, L. "L'interpretazione della costituzione tra applicazione di regole ed argomentazione basata su principî", en Rivista Internazionale di Filosofia del Diritto. 1985; ATIENZA, M., y Ruiz Manero, J. Las piezas del Derecho. Teoría de los enunciados jurídicos. Barcelona, 1996. 
7. Algunos autores suponen que la derrotabilidad de las normas y las lagunas axiológicas dependen de la presencia, en el sistema jurídico, de principios, en el sentido que serían precisamente los principios a derrotar la normas y a producir lagunas axiológicas: o, mejor dicho, los intérpretes derrotarían las normas y producirían lagunas axiológicas para aplicar uno u otro principio ${ }^{25}$. Por supuesto, a menudo esto es verdad. Pero la derrotabilidad y las lagunas axiológicas no dependen necesariamente de principios: es decir, no hay ninguna relación lógica entre estas cosas.

La derrotabilidad y las lagunas axiológicas dependen muy simplemente de las valoraciones de los intérpretes ${ }^{26}$, y a menudo esas valoraciones toman la forma de teorías jurídicas, o mejor dicho de tesis dogmáticas: algo construido por los juristas antes e independientemente de la interpretación de uno u otro enunciado normativo específico.

25 La idea de que las normas son derrotadas por los principios se encuentra, por ejemplo, en: BAYÓN, J. C. La normatividad del Derecho. Deber jurídico y razones para la acción. Madrid, 1991, p. 321 y ss.; BAYÓN, J. C." ¿Por qué es derrotable el razonamiento jurídico?", en Ídem., y RoDRíGueZ, J. Relevancia normativa en la justificación de las decisiones judiciales. p. 295 y ss.; AleXY, R. Teoría de los derechos fundamentales. Madrid, 1993, p. 98 y ss. La tesis de que las lagunas axiológicas dependen de principios ha sido defendida por RUIZ Manero, J. Ruiz Manero, J. “Algunas concepciones del Derecho y sus lagunas", en ATRIA, F., et al. Lagunas en el Derecho. op. cit.

Me parece muy ingenua la idea de RodRíGUEZ, J., según la cual la justificación de la introducción de una excepción en una norma sólo puede ser ofrecida por otra norma positiva. De hecho no es así. RodrígueZ, J. "Derrotabilidad e indeterminación del Derecho. Respuesta a JUAN CARLOS BAYÓN", op. cit., p. 240 
Es preciso señalar que las tesis dogmáticas -por ejemplo: En una república parlamentaria el jefe del Estado es un poder neutro con funciones de garantía constitucional; La costumbre internacional se forma con el consentimiento de los Estados; El ordenamiento de las Comunidades Europeas y los de los Estados miembros son ordenamientos autónomos $y$ independientes; etc.- no son enunciados interpretativos (no presentan la forma estándar de tales enunciados: El texto $T$ significa $S$ ). A veces son, muy simplemente, normas: no positivas evidentemente. Otras veces, son definiciones estipulativas que incorporan juicios de valor, preferencias políticas o morales ocultas, e implican normas. Esas definiciones orientan la interpretación y, a menudo, permiten formular normas implícitas apócrifas.

Sólo un ejemplo, por otra parte muy claro. En un fallo bien conocido -Marbury (1803)- el Tribunal Supremo de los Estados Unidos afirma que es un principio intrínseco a toda constitución escrita que un acto del poder legislativo no conforme a la constitución es nulo (a legislative act contrary to the Constitution is not law $)^{27}$. Evidentemente, esta afirmación no es un enunciado interpretativo: no hay formulación normativa en la constitución americana que pueda ser entendida en este sentido y, por otra parte, el propio tribunal tampoco intenta presentar su tesis como una conclusión interpretativa. Se trata precisamente de una tesis dogmática, que expresa directamente una norma y que permite al Tribunal sacar la norma ulterior que le otorga competencia para no aplicar las leyes inconstitucionales.

27 Tribunal Supremo de los Estados Unidos. Caso Marbury vs. Madison, 5 U. S. (1 Cranch) 137, 2 L. Ed. 60, 1803. 
8. Escribe C. AlchOURRÓN:

La derrotabilidad torna dificultosa la identificación de las normas de un sistema jurídico mucho más que la simple ambigüedad. De manera que constituye uno de los factores que hace necesario, en muchas situaciones, introducir operaciones valorativas y el uso de pautas axiológicas en la interpretación de los textos normativos ${ }^{28}$.

Estas palabras parecen suponer: por un lado, que la derrotabilidad sea una propiedad objetiva de las normas, antecedente a toda interpretación; por otro lado, que la interpretación sea -al menos normalmente- una operación que no requiere valoraciones. Las valoraciones, en el proceso interpretativo, serían no una causa, sino un efecto de la derrotabilidad.

Se trata de una concepción un poco ingenua a la vez de la derrotabilidad y de la interpretación. La derrotabilidad no preexiste a la interpretación: por el contrario, es una de sus posibles consecuencias. Y las valoraciones de los intérpretes son precisamente una causa, no un efecto de la derrotabilidad de las normas.

Así como las lagunas axiológicas no son propiedades objetivas del sistema jurídico, porque dependen de las valoraciones de los intérpretes, la derrotabilidad, igualmente, no es una propiedad objetiva de las normas (o de los enunciados normativos). No de- pende del carácter fatalmente vago del lenguaje de las

28 Alchourrón, C. E. “Sobre Derecho y lógica”, op. cit., p. 28. 
autoridades normativas: no depende de la open texture del lenguaje; tampoco depende del hecho que esas autoridades no pueden prever la infinita variedad de los casos futuros ${ }^{29}$. La textura abierta, en particular, es una propiedad objetiva e ineliminable de todos los predicados en el lenguaje natural ${ }^{30}$. La derrotabilidad en cambio -o, mejor dicho, la derrota- de las normas es el resultado de una operación interpretativa.

9. Por tanto, no tiene sentido interrogarse sobre la cuestión de si las normas jurídicas (todas o algunas) son derrotables, o no ${ }^{31}$. Las normas o, mejor dicho, las formulaciones normativas, pobrecitas, son inertes, no hacen nada: se dejan derrotar, pero no se derrotan por sí mismas. Como la hermosura no está en las cosas, sino en los ojos de quien

29 Cfr. Rodríguez, J., y SucAR, G. "Las trampas de la derrotabilidad. Niveles de análisis de la indeterminación del Derecho", en BAYÓN, J. C., y Rodríguez, J. Relevancia normativa en la justificación de las decisiones judiciales. op. cit., p. 132 y ss.; RodRíGUEZ, J. Lógica de los sistemas jurídicos. p. 364 y ss. Ambas ideas (equivocadas) se encuentran en HART, H. L. A. The Concept of Law. chapter VII. Oxford, 1961; Ídem. "The Ascription of Responsibility and Rights", en Proceedings of the Aristotelian Society. 1948, p. 49; MACCORMICK, N. "Defeasibility in Law and Logic", en Z. BANKOWSKI et al. (eds.). Informatics and the Foundation of Legal Reasoning. Dordrecht, 1995 ; SCHAUER, F. Playing by the Rules. A Philosophical Examination of Rulebased Decision-Making in Law and Life. Oxford, 1991, p. 34 y ss.; así como en CARrió, G. R. Notas sobre Derecho y lenguaje. 4. ${ }^{a}$ ed. Buenos Aires, 1994, p. 226.

30 Waismann, F. "Verifiability", en Proceedings of the Aristotelian Society. 1945, p. 19.

31 Sobre esto, sorprendentemente, parecen discutir BAYÓN, J. C., y RODRíGUEZ, J. Relevancia normativa en la justificación de las decisiones judiciales. op. cit. Cfr. también el debate contenido en los artículos de Rodríguez, J., Redondo, M. C., Caracciolo, R., Moreso, J. J., y Celano, B. Discusiones. N. ${ }^{\circ}$ 5, 2005. 
mira, la derrotabilidad no está en las normas, sino en la actitud del intérprete ${ }^{32}$.

10. La cuestión de si la derrotabilidad es una propiedad de las normas o de las formulaciones normativas es bastante simple. Una formulación normativa es un enunciado (todavía no interpretado); la derrotabilidad es una propiedad lógica; pero antes de la interpretación los enunciados no tienen propiedades lógicas: sólo las normas, en tanto significados, pueden tener tales propiedades. Entonces la derrotabilidad es una propiedad de normas, no de formulaciones normativas.

Tal vez lo que hace pensar que la derrotabilidad es una propiedad de las formulaciones normativas es el hecho que las normas que los juristas derrotan constituyen la interpretación literal de esas formulaciones: son normas prima facie $^{33}$. La falta de distinción entre un enunciado y su significado literal es cosa bastante frecuente, y esto se comprende, porque a menudo la interpretación literal consiste no en reformular, sino en reiterar sin más el texto interpretado. Sin embargo, el significado literal -incluso cuando está generalmente compartido- sigue siendo un significado, no un enunciado.

11. Hace falta distinguir cuidadosamente entre derrotabilidad, acción de derrotar, y derrota (en tanto producto de dicha acción).

32 BAYÓN, J. C. “Derrotabilidad, indeterminación del derecho, y positivismo jurídico", en BAYón, J. C., y RODRíGUEZ, J. Relevancia normativa en la justificación de las decisiones judiciales. op. cit., p. 178.

33 Tomo esta expresión de DiciotTI, E. Interpretazione della legge e discorso razionale. op. cit. 
La derrotabilidad es una propiedad disposicional de cualquier norma (en tanto interpretación literal de un enunciado normativo) $)^{34}$ : es decir, cualquier norma es diacrónicamente derrotable, ya que los juristas pueden (en sentido no deóntico, sino fáctico) derrotarla, y lo hacen continuamente ${ }^{35}$.

La acción de derrotar, en tanto proceso, es un acto interpretativo; la derrota, en tanto producto, es el resultado sincrónico de ese acto. Sincrónicamente, hay normas de hecho derrotadas (así como normas no derrotadas, por supuesto), pero no hay normas derrotables.

Una norma derrotable, mientras permanece derrotable, no sirve para nada ${ }^{36}$ : como hemos visto, no permite el refuerzo del antecedente, tampoco permite el modus ponens. Es decir, no puede ser utilizada como premisa en ningún razonamiento normativo. Por eso, los juristas sí derrotan las normas, sin embargo no las dejan derrotables hasta el infinito ${ }^{37}$. Derrotando

34 En los terminos de C. E. Alchourrón («Detachment and Defeasibility in Deontic Logic», en Studia logica, 57, 1996), cualquier norma incorpora un operador de revisión.

35 Por supuesto, como señala J. C. BAYÓN («Derrotabilidad, indeterminación del Derecho, y positivismo jurídico»), a veces la derrota de una cierta norma puede violar una "convención interpretativa» existente, o sea yo diría contradecir la «interpretación vigente» (A. Ross, 1958: On Law and Justice, London, p. 108), y por lo tanto no ser aceptada por los otros operadores jurídicos.

36 J. Rodríguez, Lógica de los sistemas jurídicos, p. 361; F. SCHAUER, Playing by the Rules, p. 116. Sea dicho de paso: la idea de HART, a menudo citada, según la cual una regla que concluye con la expresión «a menos que...» sigue siendo una regla (The Concept of Law, p. 136), me parece totalmente absurda. Tantoque dormitat Homerus.

37 J. Rodríguez y G. SuCAR, «Las trampas de la derrotabilidad. 
una norma incluyen en ella una excepción, pero esta norma, así reformulada (con alcance restringido), queda sincrónicamente inderrotable ${ }^{38}$ : apta como premisa para razonamientos en modus ponens, es decir apta para la aplicación. Y, por supuesto, diacrónicamente apta para ulteriores derrotas ${ }^{39}$.

12. Del mismo modo, también las lagunas axiológicas sólo existen de forma efímera ${ }^{40}$. Derrotando normas, los juristas crean lagunas axiológicas; sin embargo no la hacen para sostener, en serio, que el derecho es incompleto (o para sugerir a los jueces decisiones de non liquet): pues inmediatamente van a colmar las lagunas así creadas con normas nuevas apócrifas ${ }^{41}$.

Niveles de análisis de la indeterminación del derecho», p. 132.

38 En los terminos de C. E. Alchourrón («Detachment and Defeasibility in Deontic Logic»): una vez realizada la revisión del condicional derrotable, lo que queda es un condicional estricto.

39 Cfr. B. Celano, 2002: “Defeasibility” e bilanciamento. Sulla possibilità di revisioni stabili», en Ragion pratica, p. 18.

40 E. DiсіоттI, Interpretazione della legge e discorso razionale, pp. 454 y ss.

41 Un análisis de algunas técnicas de elaboración de normas «apócrifas» se encuentra en R. GUASTINI, L'interpretazione dei documenti normativi, pp. 104 y ss. 


\section{UNA OBSERVACIÓN FINAL}

¿Qué tiene que ver la derrotabilidad de las normas jurídicas con el positivismo jurídico? Nada, diría yo. Sin embargo, parece que sí: parece que admitir la derrotabilidad de las normas pueda poner en tela de juicio el positivismo jurídico $(\text { metodológico })^{42}$.

El razonamiento es más o menos el siguiente. El positivismo pretende que el derecho sea identificable independientemente de cualquier valoración moral. Pero, si las normas jurídicas son derrotables, su contenido no puede ser identificado sin valoraciones morales. Entonces el proyecto científico del positivismo está destinado al fracaso: para identificar el derecho es preciso suponer valoraciones morales.

Ahora bien, en primer lugar, este razonamiento supone evidentemente que la derrotabilidad sea una propiedad objetiva de las normas jurídicas: un problema de interpretación, y no un resultado de la interpretación. Lo que, como hemos visto, no es cierto.

En segundo lugar, este razonamiento se basa, según creo, sobre una doble confusión.

42 Cfr. una vez más la discusión entre J. C. BAYón y J. RodríGUEZ, Relevancia normativa en la justificación de las decisiones judiciales. Cfr. también M. C. REDONDO, 2006: «Tesis de relevancia y regla de clausura de la relevancia. Positivismo excluyente, positivismo incluyente, y positivismo indiferente», inédito (el paper ha sido presentado al seminario «Homenaje a Eugenio Bulygin», Barcelona: Universidad Pompeu Fabra, junio). 
1. Primera confusión. Una cosa es identificar algo -concretamente: un texto normativo- como Derecho, una cosa muy distinta es determinar su contenido normativo: qué está ordenado (permitido, prohibido), a quién, en cuáles circunstancias ${ }^{43}$. El positivismo jurídico dice simplemente que la primera de estas dos cosas se puede hacer sin valoraciones; no dice nada sobre la segunda. El positivismo metodológico no es, y tampoco incluye, una teoría de la interpretación.

La tesis positivista, en el fondo, es muy simple: cualquier mandato del soberano es Derecho. Lo que quiere decir que los mandatos del soberano no tienen que ser justos para ser Derecho: incluso las leyes injustas son Derecho. El positivismo no pretende más que esto.

En cambio, para determinar el contenido normativo de los textos normativos sí es preciso interpretar. Y esto normalmente supone valoraciones. ¿Existe alguien hoy en día que piensa, en serio, que la interpretación es una actividad wertfrei $?^{44}$.

2. Segunda confusión. Una cosa es el conocimiento científico del Derecho vigente, otra cosa muy distinta es la interpretación de los textos normativos.

Interpretar los textos normativos no es conocer el Derecho, sino contribuir a hacerlo. Si por Derecho entendemos no un

\footnotetext{
43 M. C. REDONDO, «Tesis de relevancia y regla de clausura de la relevancia».

44 Bueno, tal vez sí: esto parece ser el caso de R. HERNÁNDEZ Marín, 1999: Interpretación, subsunción, y aplicación del Derecho, Madrid-Barcelona.
} 
conjunto de enunciados, sino un conjunto de significados, no hay Derecho sin interpretación: el Derecho resulta de una combinación de legislación (en sentido material: emisión de formulaciones normativas) y de interpretación. Entonces, la interpretación no coincide con la identificación del Derecho. Más bien la interpretación es parte del Derecho: un aspecto del objeto que se quiere identificar o conocer.

Dicho de otra forma, la interpretación no es la ciencia del derecho, sino que forma parte de su objeto. La ciencia jurídica es un discurso de segundo nivel (un metalenguaje) respecto al discurso interpretativo. 